\title{
ArcheoSciences
}

Revue d'archéométrie

41-2 | 2017

Varia

\section{La polarisation provoquée, outil géophysique de spatialisation des amas de scories pour l'estimation des productions sidérurgiques}

Induced Polarization: a geophysical tool for slag characterization and ancient iron production assessment

Nicolas Florsch, Abdulsamad Feras, Jeremy Bonnenfant et Christian Camerlynck

\section{OpenEdition}

\section{Journals}

Édition électronique

URL : https://journals.openedition.org/archeosciences/4958

DOI : 10.4000/archeosciences.4958

ISBN : 978-2-7535-7393-2

ISSN : $2104-3728$

Éditeur

Presses universitaires de Rennes

Édition imprimée

Date de publication : 8 décembre 2017

Pagination : 23-33

ISBN : 978-2-7535-7391-8

ISSN : 1960-1360

Référence électronique

Nicolas Florsch, Abdulsamad Feras, Jeremy Bonnenfant et Christian Camerlynck, « La polarisation provoquée, outil géophysique de spatialisation des amas de scories pour l'estimation des productions sidérurgiques », ArcheoSciences [En ligne], 41-2 | 2017, mis en ligne le 08 décembre 2019, consulté le 01 février 2022. URL : http://journals.openedition.org/archeosciences/4958 ; DOI : https://doi.org/

10.4000/archeosciences.4958 


\title{
La polarisation provoquée, outil géophysique de spatialisation des amas de scories pour l'estimation des productions sidérurgiques
}

\author{
Induced Polarization: A Geophysical Tool for Slag Characterization \\ and Ancient Iron Production Assessment
}

\author{
Nicolas FlorsCh ${ }^{a}$, Abdulsamad Feras ${ }^{b}$, Jeremy Bonnenfant ${ }^{c}$ \\ et Christian Camerlynck ${ }^{\mathrm{b}}$
}

À la mémoire d'Alain Ploquin

\begin{abstract}
Résumé : Estimer l'ampleur d'une ancienne exploitation minière profite à l'historien dans sa recherche de quantification des richesses issues des matières premières. S'agissant du fer, c'est aussi du pouvoir militaire dont il peut s'agir. Mais en l'absence d'archives préservées, il faut en passer par l'archéologie car alors seuls les vestiges du terrain permettent une estimation de la production de métal. Les méthodes géophysiques, prises comme outils de cartographie des amas de scories, peuvent contribuer à la compréhension d'un site métallurgique. Toutefois, les méthodes classiques, magnétiques, électriques et électromagnétiques ne sont pas des proxys directs des volumes de scories abandonnées. Elles ne fournissent pas une réponse spécifique et ne sont pas conservatrices (la réponse intégrale n'est pas proportionnelle à l'intégrale des masses ou volumes). Par contre, la méthode de Polarisation Provoquée (PP), d'ailleurs traditionnelle pour la recherche minière depuis le milieu du xx siècle, offre, pour les amas de scories, une réponse spécifique, linéaire, conservative. Nous l'utilisons au lieu-dit "Grand Bois ", sur la commune de Saint-Vincent-sur-l'Isle (Dordogne, France), pour un site d'extraction actif du XI ${ }^{\mathrm{e}}$ au Xve siècle. La PP nous permet de caractériser la géométrie de l'amas de scories et par suite rend possible l'estimation du volume de fer produit sur la plateforme métallurgique majeure du site étudié.
\end{abstract}

\begin{abstract}
Assessing the importance of an ancient iron production site benefits to the historian in his research about authorities' wealth and strategies, including military needs. When preserved archives are lacking, archaeology is a mandatory framework, and only site artefacts allow for production assessment. Applied geophysical methods, as tools for slag heap characterization, help to understand site spatial organization. However, magnetic and electromagnetic methods do not preserve means, and do not lead to conservative estimators. Conversely, Induced Polarization (IP), which is by the way widely used in mining research since the middle of the 20th century, offers a specific and linear response. We use it on the "Grand Bois" (Saint-Vincentsur-l'Isle, Dordogne, France) where it allows characterizing the shape of the slag heaps, and finally leads to a suitable iron ancient production.
\end{abstract}

Mots clés : Polarisation Provoquée, paléométallurgie, amas de scories, Saint-Vincent-sur-l'Isle.

Keywords: Induced Polarization, ancient metallurgy, slag heaps, Saint-Vincent-sur-l'Isle.

\footnotetext{
a Sorbonne Universités, UPMC université Paris 6, IRD, UMI 209, UMMISCO, Bondy, France.

' Sorbonne Universités, UPMC université Paris 6, CNRS, UMR 7619, METIS, Paris, France.

${ }^{c}$ CNRS, UMR 5607, AUSIONUS, France.
} 


\section{INTRODUCTION}

\section{Problématique archéologique}

Le volume de métal fer produit sur un site ou une région donnée sur une période donnée constitue un indicateur économique important de l'activité industrielle, quels que soient les époques et systèmes de gouvernance auxquels cette activité peut être rattachée.

S'agissant de sites d'ampleur, le fer produit est fréquemment transporté hors des districts miniers, vers les lieux de son utilisation. L'estimation des volumes extraits de minerai est difficile par définition, ces volumes ayant disparu, ce qui rend subséquemment l'estimation du métal produit difficile.

Le chercheur se convainc rapidement qu'un des meilleurs "proxy " possible du volume de fer produit est accessible par l'intermédiaire du volume des scories rejetées sur le site. C'est la connaissance de coefficients de conversion obtenus lors d'études antérieures qui permet cette conversion, comme le montre l'exemple de Decombeix et al. (1998).

Le plus souvent, pour des sites non remaniés par des travaux contemporains, l'archéologue a de bonnes raisons de penser que les amas de scories sont préservés. Le facteur de conversion en fer produit dépend du type de minerai et va varier selon le district, tout en restant valable pour un ensemble donné, dès lors que l'on peut disposer d'un estimateur de ce coefficient. L'archéologie expérimentale est une des approches fécondes et alternatives à des recherches plus traditionnelles, qui permet de consolider l'estimation de ce rendement. Parallèlement, une cartographie (ou spatialisation) des scories contribue également à comprendre l'organisation opérationnelle d'un site d'ateliers, et peut apporter un éclairage complémentaire sur la chaîne de traitement.

Les amas de scories résultent d'un démantèlement continu ou discontinu des coulées obtenues à la base des fourneaux (Jarrier et al., 1997; Mahé-Le Carlier et Ploquin, 1999; Fluzin et al., 2000). Ils présentent un granoclassement hétéroclite, allant de blocs pluridécimétriques à de la poussière, et la masse volumique macroscopique des amas est souvent voisine ou inférieure à $2000 \mathrm{~kg} / \mathrm{m}^{3}$ en raison d'une forte proportion de vides entre les blocs les plus volumineux. La densité est également déterminée par la proportion, parfois importante, de bulles.

Le site archéologique faisant l'objet de la présente étude se situe en Dordogne à une quinzaine de kilomètres de Périgueux, au lieu-dit "Les Grands Bois » sur la commune de Saint-Vincent-sur-l'Isle (voir l'annexe). Il comporte plusieurs ateliers sidérurgiques : on y dénombre onze ferriers, ciblant les lieux où la production du fer s'organisait. Dix d'entre eux se situent dans la partie sud-ouest du site et un (le numéro 10) est à environ deux kilomètres à l'est du numéro 1. La production de fer de ce petit ferrier isolé à l'est est supposée être la plus ancienne, datant du XI siècle. Les autres auraient eu une activité plus récente, datée de la deuxième moitié du XII ${ }^{\mathrm{e}}$ siècle jusqu'à la première moitié du Xv siècle (Bonnenfant, 2015a). Sur le ferrier le plus important, il existe des traces d'un bâtiment. Les fouilles archéologiques ont permis de mettre au jour divers mobiliers domestiques : pots, marmites et des plaques foyères (Bonnenfant, 2015b). Le bâtiment correspondrait à un espace dédié à la vie des ferrons sur leur lieu de travail. On sait que la production de fer brut à cette époque se faisait notamment à l'aide de bas fourneaux, lesquels sont seuls attestés sur ce site. La réduction, qui ne nécessitait pas que le métal fût porté à la fusion, conduisait à une « loupe de fer ", que le métallurgiste expurgeait des restes de charbons de bois et d'autres impuretés, par un martelage technique : le cinglage. Actuellement en forêt, le ferrier principal couvre environ $2600 \mathrm{~m}^{2}$. Il se situe à 210 mètres d'altitude, au sommet d'un plateau constitué d'une poche sidérolithique. Il est encadré par deux rivières : l'Isle et l'Auvezère. Au nord de ce ferrier, et juste à côté, une grande zone a été défrichée par le propriétaire en 2014, détruisant potentiellement des vestiges d'atelier et d'extraction.

Pour ce site de Dordogne qui nous concerne dans ce travail, on observe une interface entre les couches de scories et le terrain sous-jacent particulièrement franche. Même pour les sites les plus anciens, la couche de scories n'est pas mélangée au sol, pour la simple raison que le sol sus-jacent est souvent absent, ou très peu développé. À noter que ces scories présentent une perméabilité en grand telle que rien ne retient longtemps l'eau en surface là où elles sont présentes. La situation est ainsi très différente de la plupart des sites de montagne, où les scories sont mêlées au tout-venant du colluvionnement ou ont fait l'objet d'une remobilisation. Dans ce dernier cas, l'obtention d'un estimateur nécessite la spatialisation de la proportion de scories au sein du milieu remobilisé par des causes naturelles ou anthropiques.

Le ferrier 1 qui nous intéresse étant le plus grand, il est aussi le plus représentatif de la production métallurgique de l'époque. Il occupe un terrain marqué par un fort relief, avec des ondulations de longueur d'onde allant de 3 à 15 mètres et ayant une amplitude maximale de 3 mètres. La photo de la figure 1 montre clairement la disposition des scories sur le substratum sidérolitique. Le mur de la couche de scorie elle-même est fortement ondulée. La brutalité de la transition détermine des réponses géophysiques très franches, la polarisation provoquée répond en effet avec un signal fort sur les scories, mais faible sur la couche sidérolitique (malgré la présence de nodules ferrugineux). 
Figure 1: (Voir planche couleur VII) La limite entre les scories et le substratum apparaît bien, avec une transition brutale. Figure 1: (See colour plate VII) The interface between the slag layer and the siderite substratum is clearly defined on the field.

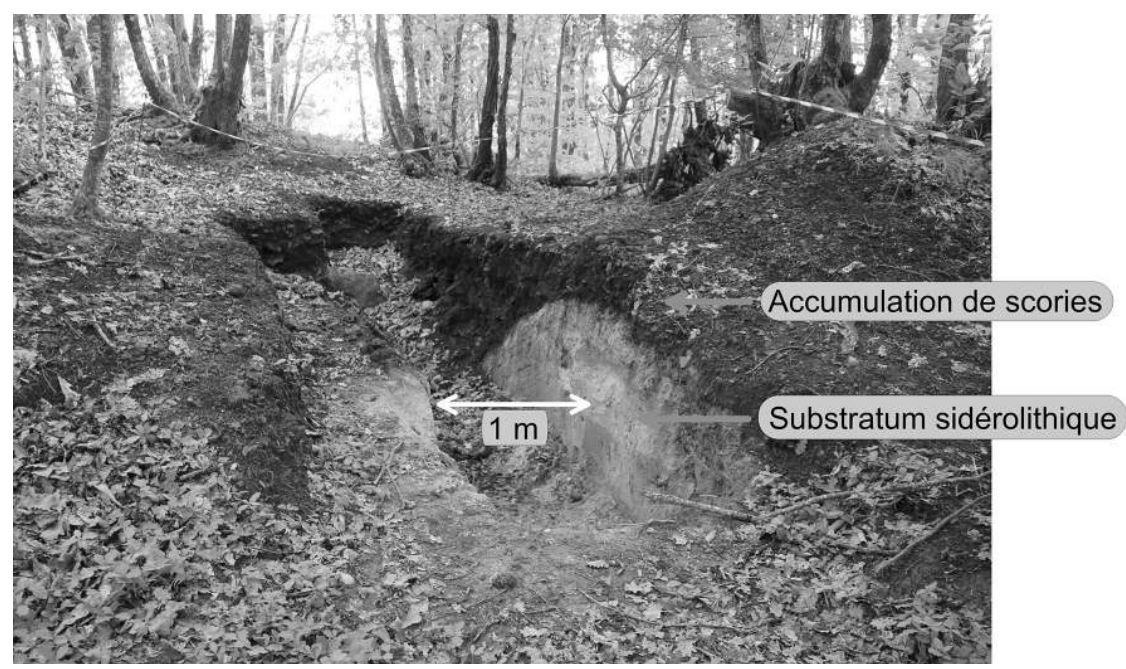

Il n'existe aucune mention de ce site dans les archives. Les recherches sont donc menées pour comprendre son fonctionnement et pour atteindre in fine une évaluation de l'importance du site du point de vue historique. Avant les opérations de prospection géophysique, l'estimation du volume de scories est de $1585 \mathrm{~m}^{3}$ ce qui est indicateur d'une grosse production de fer et donc d'une exportation au niveau régional ou plus. Néanmoins, ce volume est estimé à partir de l'enveloppe du ferrier en surface et de la base de la couche de scories, telle qu'elle a pu être échantillonnée dans les sondages archéologiques, ces derniers ne pouvant donner que des informations ponctuelles. La géophysique a justement pour but d'obtenir une estimation plus précise de ce volume.

\section{La Polarisation provoquée}

La méthode PP peut être vue comme une extension de la méthode électrique à courant continu, dont elle emprunte les principes géométriques et protocolaires de terrain. Mais tandis que cette dernière concerne des courants galvaniques continus - donc à fréquence nulle -, la PP explore un spectre de fréquences allant de quelques milliHertz $(\mathrm{mHz})$ à quelques kiloHertz $(\mathrm{kHz})$. Deux gains résultent de cette extension. D'une part, les spectres, sur ce domaine de fréquences, peuvent dépendre de 2 à 5 paramètres qui sont autant de degrés de liberté (contre un seul, la résistivité, en courant continu). D'autre part, la physique en jeu n'est pas la même : elle porte sur des phénomènes de polarisation électrique (Revil, 2013).

Les théories associées à cette méthode sont en pleine évolution, grâce à la mobilisation d'une communauté active à l'échelle internationale. La théorie classique est synthétisée dans les deux tomes de Bertin et Loeb (1976), tandis que cette dernière décennie, les chercheurs ont organisé des colloques dédiés, dont le dernier est référencé sur la toile [http:// hgg.au.dk/ip2016/] où de nombreuses présentations peuvent être téléchargées (IP2016, 2016).

Le phénomène de polarisation est universel. Il est relatif à la capacité des roches et des sols à concentrer, de manière réversible, des éléments chargés, (ions, électrons) au sein des milieux pouvant contenir de telles charges et particulièrement au voisinage des grains constitutifs des matériaux naturels. En effet, dans les milieux naturels contenant des électrolytes et donc des cations et des anions, un courant injecté dans le sol au moyen de deux électrodes provoque une redistribution spatiale des charges positives et négatives, laquelle dépend également du temps. Dans les scories, les particules à conductivité électronique (magnétite, certains sulfures) présentent une forte polarisabilité, propre aux matériaux à conductivité électronique. Décrivons succinctement le principal mécanisme en jeu, le lecteur pouvant se référer, pour la théorie détaillée, aux récents articles de Revil et al. (2015a, 2015b).

\section{Mécanisme de la polarisation provoquée}

Les particules à conductivité électronique présentent une conductivité très élevée par rapport à la conductivité de l'encaissant. Selon Olhoeft (1981), pyrite et galène présentent des résistivités de l'ordre de $0,001 \Omega \mathrm{m}$ et même 10 fois moins pour la magnétite. Une nouvelle répartition des charges intervient alors au sein de ces particules. Ce sont, en quelque sorte, ces déséquilibres de charge que l'on cherche à mesurer. Dans un encaissant qui est pratiquement un million de fois plus résistant, au temps près, très court, de migration des électrons et trous d'électrons dans la particule, cette dernière est pratiquement équipotentielle; ou encore, 
le champ électrique total à l'intérieur de la particule s'annule. Ainsi, les charges mobiles dans la particule s'organisent de sorte à compenser exactement le champ électrique primaire. $\mathrm{Vu}$ de l'extérieur de la particule, cette dernière devient un petit dipôle électrique, qui porte une double influence sur son voisinage, selon que l'on considère ce qui se passe dans la double couche électrique (à moins de $20 \mathrm{~nm}$ de la surface de la particule) ou plus loin, à une distance de l'ordre du diamètre de la particule. Dans cette couche proche qui s'apparente à une couche de Stern non permanente, il apparaît une migration des charges externes (les ions) qui répondent aux charges internes (électrons et trous), l'équilibre s'établissant en raison du carré du diamètre du grain et en raison inverse $\mathrm{du}$ coefficient de diffusion des ions dans la couche. Dans cette couche, l'on peut dire que les forces intervenant sont surtout des forces de Coulomb et des forces de viscosité. Au-delà de la double couche électrique, les ions de la solution répondent également à la sollicitation électrostatique du dipôle, mais dans un contexte de compétition entre l'autoorganisation électrostatique et une tendance à la dispersion thermique (brownienne). Les équations qui rendent compte de l'ensemble du phénomène sont dites de Poisson-NernstPlanck et sont non-linéaires, ce qui complique les approches théoriques (voir par exemple Zheng et al., 2011). Toutefois, des modélisations numériques récentes et adaptées à la PP rendent assez bien compte de ces phénomènes microscopiques (Abdulsamad et al., 2016).

À l'échelle du terrain, le récepteur mesure la somme des effets de tous les dipôles microscopiques. Trois modes expérimentaux peuvent être mis en œuvre :

- L'approche temporelle, avec un courant émis qui est brutalement coupé, suivi d'un temps d'écoute où l'on peut observer la dépolarisation du milieu, comme l'ont observé les découvreurs de la PP en 1920 (Schlumberger, 1920). En première approximation, on observe une décroissance exponentielle de la tension, avec une certaine amplitude et une constante de temps caractéristique (une fraction de secondes à quelques secondes).

- L'approche "fréquentielle ", importante dans l'histoire de la prospection minière, où le courant est injecté en alternatif rectangle, à deux fréquences différentes choisies entre 0.1 et $10 \mathrm{~Hz}$.

- L'approche spectrale, où toute la fonction de transfert fréquentielle est presque continûment explorée entre $0,001 \mathrm{~Hz}$ et $10 \mathrm{kHz}$ (typiquement).

La progression de la méthode a suivi l'évolution des techniques de mesure, de plus en plus fines et automatisées. Initialement, et jusqu'à la fin du $\mathrm{Xx}^{\mathrm{e}}$ siècle, les cibles de la $\mathrm{PP}$ sont celles qui produisent un très fort signal (une très forte chargeabilité). Ce sont principalement des gisements miniers de sulfures disséminés, pour lesquelles non seulement la méthode excelle, mais est même souvent exclusive. En ce $\mathrm{XxI}^{\mathrm{e}}$ siècle, et suite aux progrès instrumentaux, la PP spectrale est devenu possible, et les progrès concomitants de l'électronique permettent d'appréhender les signaux faibles de cibles environnementale (Kemna, 2000; Kemna et al., 2012). Ainsi utilise-t-on la PP, entre autres exemples, pour la délimitation de pollutions par exemple aux hydrocarbures, comme dans Kemna et al. (2004), ou encore pour la détermination de la perméabilité (Revil et Florsch, 2010).

Pour les deux premières méthodes temporelle et fréquentielle, le lecteur peut se référer par exemple à Telford et al. (1990), ou à Parasnis, (1997). En ce qui concerne la polarisation provoquée spectrale, on pourra se référer par exemple à la thèse de Sheen (2016), qui constitue une synthèse. Seigel et al. (2007) proposent une histoire résumée de la méthode, qui toutefois n'intègre pas les dernières évolutions.

Nous avons utilisé ici l'approche temporelle classique, notamment pour sa rapidité de mise en œuvre sur le terrain et son efficacité sur les milieux bien polarisables (ce qui est le cas des scories). Le courant injecté est pratiquement une alternance de courants continus dans les deux sens, avec un créneau d'écoute, sans injection, durant lequel le potentiel, forcément secondaire et de dépolarisation, est enregistré. L'alternance permet une compensation de la "polarisation spontanée».

Le principal paramètre retenu est l'intégrale de la courbe de décroissance, que l'on nomme la " chargeabilité ». Selon le type de normalisation, la chargeabilité est sans unité (souvent en \%o ou en $\mathrm{mV} / \mathrm{V}$ ) ou en milli-seconde (ms) (pour une utile discussion sur cette question, voir notamment Mao et al. [2016]). La figure 2 illustre les alternances de courant et le signal mesuré.

Lappareil utilisé sur le terrain est un Syscal $\operatorname{Pro}^{\circledR}$ de Iris Instrument. Nous avons utilisé des créneaux d'injection de 1 seconde (période totale $4 \mathrm{~s}$ ), avec $200 \mathrm{~V}$ au générateur, et mesuré l'intégrale de la courbe de décroissance entre $20 \mathrm{~ms}$ et $850 \mathrm{~ms}$ après les coupures du courant. Les mesures sont répétées pour être accumulées ("stackées ») entre 3 et 6 fois, avec un contrôle qualité (répétabilité) pour être meilleur que $0,5 \%$.

Dans le domaine de la paléométallurgie et de la paléosidérurgie, plusieurs travaux antérieurs valident l'utilisation de la PP pour la détection et la caractérisation géométrique des amas de scories: Weller et al. (2000) ; Ullrich et al. (2007), Meyer (2007); Florsch et al. (2011 et 2012) : PlacenciaGómez et al. (2015). Signalons que la polarisation provoquée est également possible sur des bois anciens (Schleifer et al., 2002) : son champ d'application archéométrique déborde du seul cas métallurgique, et les applications de 
Figure 2 : Signaux mis en jeu en polarisation provoquée. En haut, le courant alternatif, de quelques centaines de milli-ampère, est constitués de créneaux de durée choisie $(0,5$ à 2 secondes typiquement). Au milieu, la tension (réduite de la polarisation spontanée des électrodes) montre le retard au chargement et au déchargement.

Figure 2: Involved signals for Induced Polarization. On the top, the current is a classically between a few $m A$ and one Amp, and is made of a successive positive, null, negative, and null current segments, lasting from 0.5 to $2 \mathrm{sec}$. The bottom shows the resulting potential, with a magnified detail of the observed segment where the decay is under study.
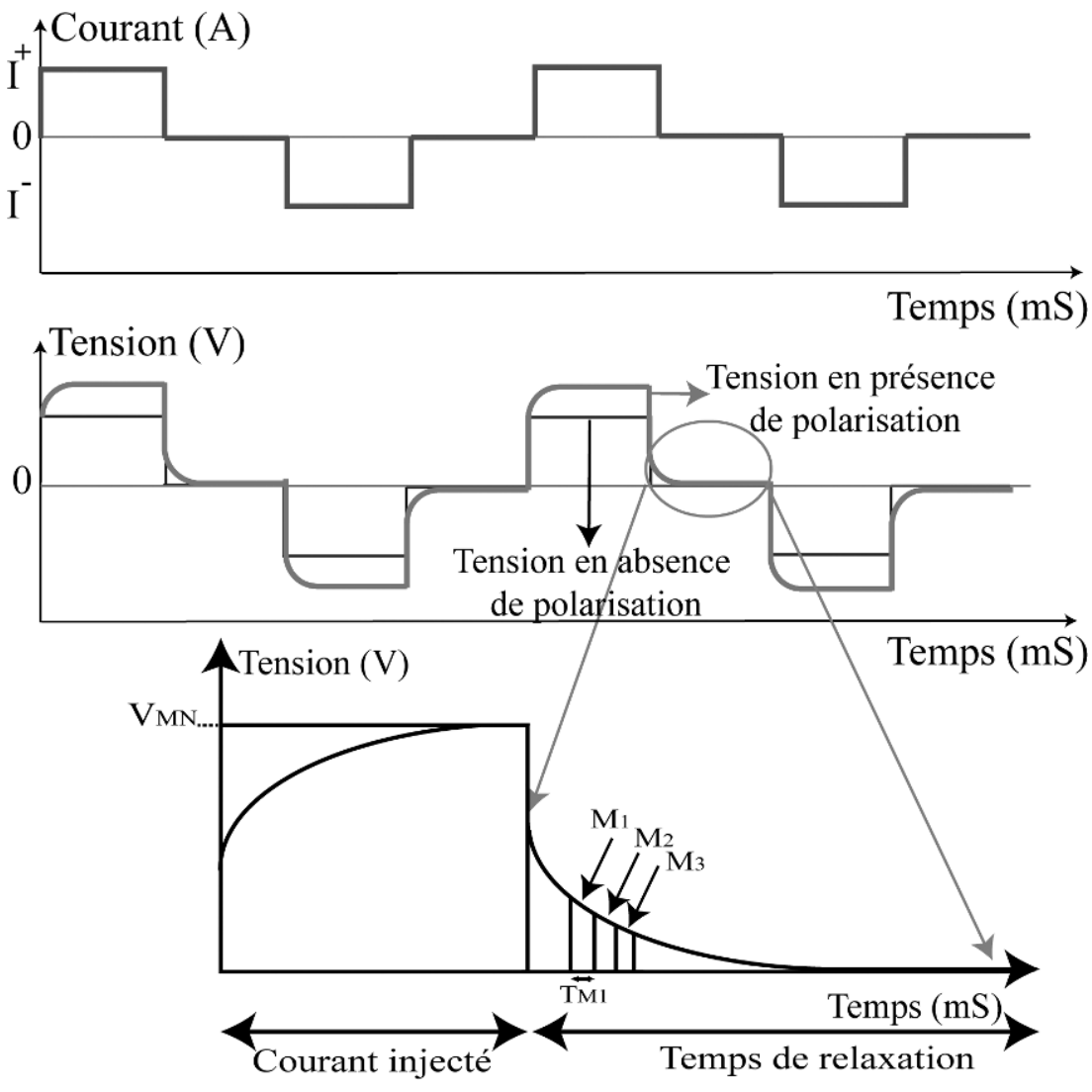

prospection par PP dans le domaine de l'archéométrie en sont certainement à leurs débuts.

\section{Matériel et méthode}

Les dispositifs géométriques utilisables pour la PP sont les mêmes que pour les méthodes électriques; on retrouve les protocoles de "traînée " (cartographie 2-D), de tomographie 2-D (souvent dénommés " panneau électrique » en France) et 3-D, de sondage électrique.

Pour la tomographie, les électrodes, pré-positionnées, sont connectées au générateur et au voltmètre ad hoc selon une séquence définie par avance. À deux dimensions (une horizontale, l'autre verticale), les différentes combinaisons explorent les résistivités apparentes dans l'axe du profil et pour différentes profondeurs. C'est là le principe habituel de la tomographie électrique, étendue ici en " résistivité complexe ». Ainsi, la PP spectrale (qui est la méthode la plus générale) revient à prendre en compte les composantes en phase et en quadrature, en fonction de la fréquence, d'une résistivité rendue complexe (au sens des nombres imagi- naires). Les présentations de Loke (à télécharger sur http:// www.geotomosoft.com/), également «PI " du programme d'inversion que nous avons utilisé (RES2DINV, Loke, 2002; Loke et Barker, 2004), constituent une bonne introduction concernant la tomographie de résistivité électrique et de polarisation provoquée.

\section{Implantation des mesures sur le terrain et protocole de mesure}

Une section de mesures comporte ici 48 électrodes en ligne, espacées de $1 \mathrm{~m}$. Pour les résultats de mesure, le géophysicien utilise la "pseudo-section", terme relatif au mode de représentation en fonction de l'offset le long du profil et en fonction de la profondeur d'investigation. En effet, les mesures sont reportées, en $\mathrm{x}$ horizontalement et, en fonction de la profondeur, verticalement, selon un « $\mathrm{z}$ " conventionnel, (" pseudo-z ») choisi pour rendre commode la représentation des données brutes. L'image à deux dimensions $(\mathrm{x}, \mathrm{z})$ obtenue est appelée "pseudo-section ». Afin de reconstituer des résistivités " vraies ", les données alimentent un programme d'inversion. Ce dernier fournit en sortie une 
" section de résistivité » qui reproduit la réalité, avec toutefois certaines limites de résolution et d'unicité. En réalité, le programme d'inversion fournit une solution (une section de résistivité), donc une répartition selon $\mathrm{x}$ et $\mathrm{z}$, telle que la pseudo-section que l'on peut déduire par calcul de ce modèle ressemble bien aux mesures (souvent à mieux que $3 \%$ près).

Il est important de lister les limitations de cette technique d'imagerie très répandue $(\mathrm{ERT}=$ Electrical Resistivity Tomography en anglais).

- Dans un tel processus, un modèle 2-D est mis en œuvre, tandis que la réalité est 3-D (il existe une tomographie 3-D, mais son apport relatif s'inscrit dans une recherche de compromis efficacité-coût).

- La limite de résolution est fixée par la nature de la physique mise en jeu; à moins d'hypothèses fortes sur le modèle, la résolution est de l'ordre du quart de la profondeur (ceci restant subjectif selon la répartition des résistivités dans le sous-sol).

- En aucun cas la solution n'est unique. Celle affichée résulte de choix algorithmiques et d'a priori dont le moindre n'est pas que l'on recherche une solution lisse (sur la base que la résolution est de toute manière limitée).
Au total, 15 pseudo-sections sur une base de 32 électrodes ont été réalisées, numérotées P0 à P14. La P14 ne comporte que 24 électrodes car elle se trouvait en bordure de terrain et en partie sur la route. La section P0 est en extrémité du site, sur la partie qui a été défrichée. Elle peut servir, à ce titre, de référence de signal nul, en quelque sorte, car aucun signal PP n'est attendu sur cette section qui se situe en dehors de la zone à scories (ce qui est confirmé par la pseudo-section obtenue, non présentée ici).

La figure 3a montre l'implantation géoréférencée en système de coordonnées Lambert 93 de ces pseudos-sections, en superposition avec la topographie locale.

Le dispositif utilisé est le Wenner-Schlumberger réciproque, avec les électrodes de réception $(\mathrm{M}$ et $\mathrm{N})$ aux extrémités, et les électrodes d'injection (A et B) de part et d'autre du milieu de MN. L'utilisation d'un dispositif réciproque (inversion des couples $(\mathrm{AB}$ et $\mathrm{MN}$ ) permet une parallélisation qui conduit à un gain significatif de la durée d'obtention d'une séquence complète de mesures. Chaque pseudo-section comporte environ 600 mesures. Les électrodes utilisées sont en acier. Nous avons également testé des électrodes impolarisables $\left(\mathrm{Cu} / \mathrm{CuSO}_{4}\right)$. Elles permettent de réduire le bruit d'un facteur 2, ce qui est (a) Topographie

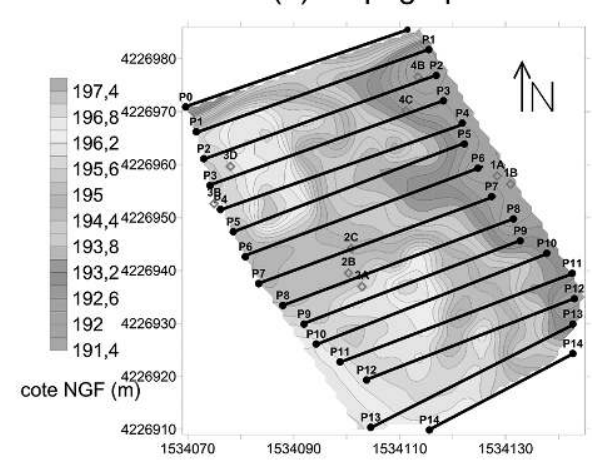

(c) Epaisseur de la couche de scories

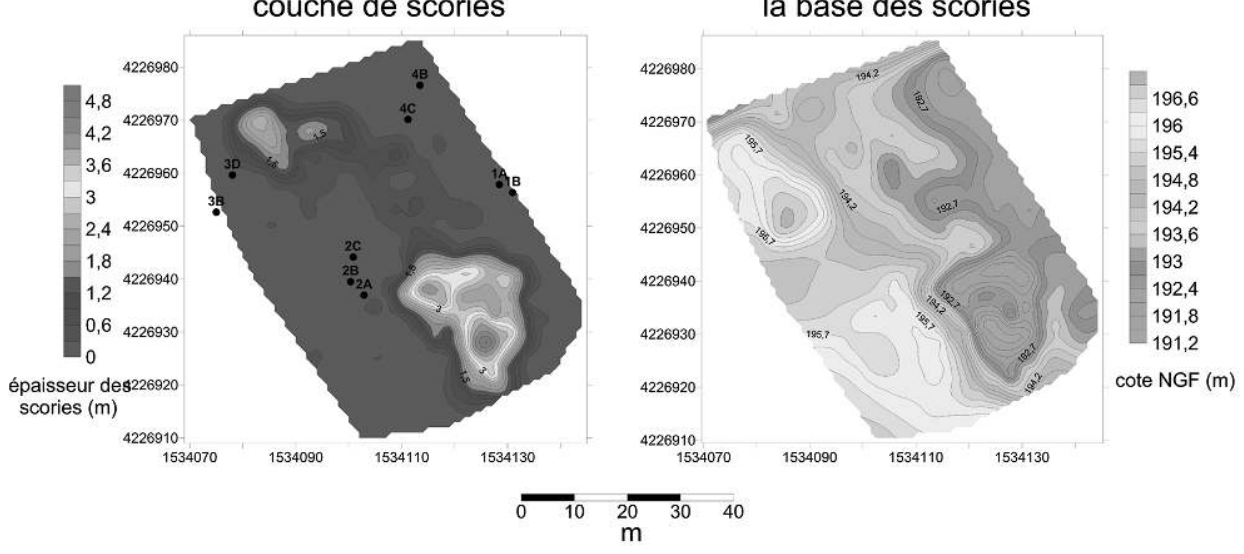

Figure 3: (Voir planche couleur VIII) Les cartes sont géoréférencée en Lambert 93 (RGF93), avec la cote NGF en mètre. a) Topographie et implantation des sections de résistivités (traits noirs). Les carrés rouges sont des repères topographiques; b) Topographie en perspective (grille blanche) drapée en 2-D de l'épaisseur des scories; c) Epaisseur de la couche de scories (toujours affleurante); d) Topographie de la base (ou mur) de la couche de scorie). On observe que l'amas principal fait relief en surface, mais occupe une zone en creux (artificielle ou pré-existante). Figure 3: (See colour plate VIII) Georeferenced maps (Lambert 93 [RGF93]). a) topographic surface with 2-D ERT profiles; b) perspective topographic surface (white grid) with draped 2-D slag layer thickness (in color); c) slag layer thickness obtained by compiling the chargeability tomography slices; d) slag layer bottom topography. 
a priori notable. Mais ce gain n'opère que lorsqu'une électrode venant d'être utilisé en émission sert tout de suite après en réception : l'électrode reste bien polarisée quelques temps. En arrangeant le protocole de sorte à éviter ce cas de figure, quelques points sont perdus, mais le bilan final indique que l'on gagne, sur une durée donnée, bien plus de points avec les électrodes en acier, car leur implantation est notablement plus rapide.

Pour parfaire les résistances de prise de terre et optimiser le travail, les électrodes ont systématiquement été arrosées avec une eau légèrement salée, puis laissées quelques minutes au repos, avant de lancer les séquences de mesures.

\section{Topographie}

Le relief est localement important (Figures 1, 3a et 3b). Les électrodes sont implantées tous les mètres, les intervalles étant pris horizontalement (et non pas selon l'abscisse curviligne le long des pentes). Leurs positions en ( $\mathrm{x}, \mathrm{y}, \mathrm{z})$ sont relevées au théodolite. Le relief le long du profil modifie la répartition du courant dans le sol, et le programme d'inversion RES2DINV prévoit la prise en compte du relief de sorte à en corriger les principaux effets, qui sont loin d'être négligeables. Les effets latéraux du relief sont toutefois limités (mais non nuls), parce que le dispositif et les courants suivent une même direction (celle du profil).

\section{Exemple de section et utilisation}

La figure 4 montre les sections P12, en chargeabilité et résistivité respectivement. Une certaine corrélation existe, elle est inhérente au fait que les scories présentent un contraste avec l'encaissant tant en chargeabilité qu'en résistivité. Toutefois, le contraste de résistivité n'est pas discriminant : il existerait tout aussi bien avec un amas de pierres résistant, tandis que la réponse en PP ne peut avoir lieu que lorsque le matériau est effectivement polarisable.

Les questions de " co-gestion ", en quelques sortes, des données de PP avec les données de résistivité font débat, en raison des corrélations apparentes ou réelles qui peuvent exister, selon que l'on s'intéresse à un signal associé à des particules métalliques ou non. Mao et al. (2016) offrent une synthèse pertinente sur cette question, et proposent que, dans les cas de particules métalliques (entendre : à conductivité électronique), il ne soit pas fait de normalisation particulière.

\section{Conversion en épaisseur de scories et cartographie de cette épaisseur}

Notre objectif principal est d'estimer le volume de scories en présence. Cela doit être entrepris dans un contexte où les sondages archéologiques révèlent une couche de scories homogène mais d'épaisseur très variable recouvrant le sidérolitique : c'est du " tout ou rien ", et il n'est pas observé, dans la couche contenant les scories, de variation significative de concentration de ces dernières. La transition brutale se fait en quelques centimètres, comme cela est bien illustré sur la figure 1 .

En conséquence, calculer le volume de scories revient à calculer le volume compris entre la surface et la base des scories. Cette démarche est différente de celle utilisée par Florsch et al. (2011) dans laquelle une relation affine entre la chargeabilité et la concentration de scories, relation calibrée en laboratoire, était utilisée, mais pour cette dernière étude, les scories étaient davantage mélangées au sol d'origine.

Pour la détermination de l'interface (la base des scories), nous avons utilisé une idée simple, appliquant en cela une sorte de rasoir d'Ockham. Nous avons considéré que l'interface se situe, pour chaque section, sur l'iso-valeur corres-
Figure 4 : (Voir planche couleur VIII) Résistivité (en haut) et chargeabilité (en bas) de la section 12 , après inversion RES2DINV.

Figure 4: (See colour plate VIII) Resistivity (top) and chargeability (bottom) relative to profile 12, after RES2DINV inversion.
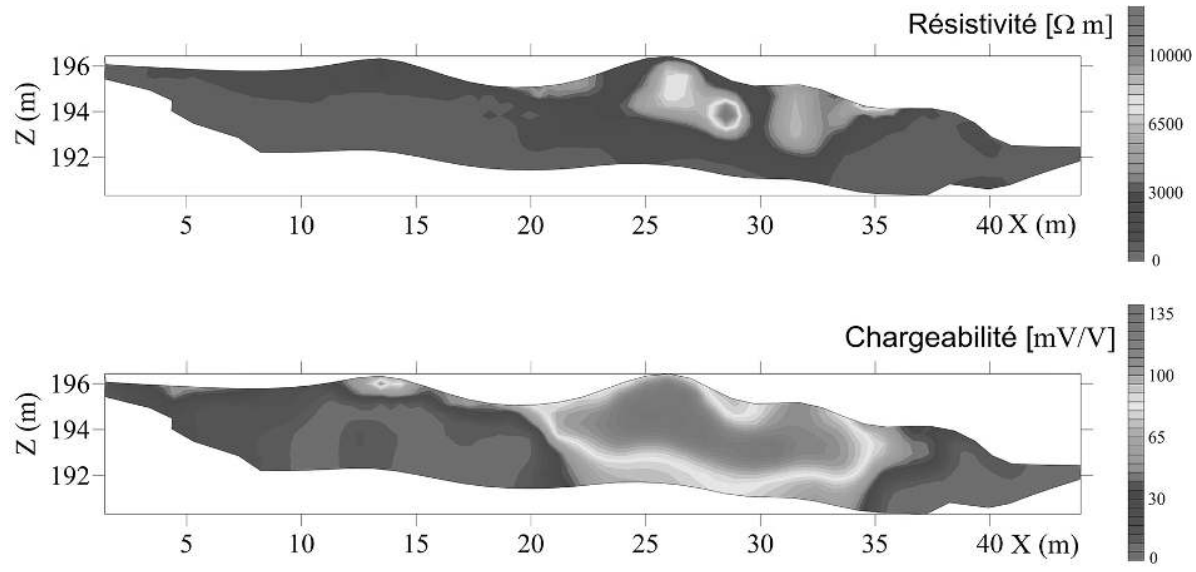
pondant à la moitié de la valeur maximum de chargeabilité rencontrée sur la section. Une justification possible de cette règle repose sur la linéarité de la réponse $\mathrm{PP}$ par rapport à la concentration de scorie, en prenant en compte la concentration en tout ou rien : ou bien $100 \%$ de scories, ou bien $0 \%$.

Nous avons cherché les limites de ce raisonnement via des modélisations. Nous avons observé que la limite ainsi définie n'est pas réellement distinguable en pratique du point d'inflexion de la courbe de décroissance de la chargeabilité avec la profondeur, et que la méthode est validée même en présence de forts contrastes de résistivité.

Il est pertinent ici de faire une remarque générale concernant cette propriété de linéarité de la réponse PP, linéarité "par rapport aux structures». Elle n'existe pas en résistivité, si bien qu'une estimation de teneur en eau, par exemple, via la loi d'Archie, ne peut être qu'erronée (sauf si l'on ne considère que de faibles variations, auquel cas il est possible de linéariser localement, c'est-à-dire de considérer les anomalies comme des perturbations). La méthode électrique «bute » en effet dans les extrémités de ses lois d'équivalence : la moyenne n'est généralement pas conservée. Prenons l'exemple d'un objet conducteur enfoui, de conductivité qui serait infinie, dans un encaissant classique : les résistivités apparentes et inversées ne permettraient pas de recouvrer cet infini. Ainsi, tandis que la moyenne des conductivités est infinie (puisqu'il existe un sous-domaine où la conductivité est infinie), les conductivités reconstituées après inversion sont bien à moyenne bornée. De la même manière, une cavité, de résistivité infinie, ne peut être détectée que comme un bon résistant.

La polarisation provoquée ne présente pas ces limitations, et offre une bonne linéarité. Cette propriété peut être vue comme résultant du fait que la PP peut, elle, être présentée comme une perturbation du problème électrique, où l'on adjoint, à la conductivité classique, un terme de perturbation imaginaire pur et petit.

Enfin, un argument sur la linéarité provient des résultats d'autres campagnes, notamment celles faite en Ariège (France) sur le site d'Aulus-les-Bains et présentée dans Florsch et al. (2012). La masse de scories estimée à partir de la géophysique a pu être comparée à celle effectivement excavée. L'écart était de $6 \%$. Cependant, en effectuant une modélisation suivie d'une inversion, l'écart a pu atteindre $20 \%$, ce qui est moins bon. La cause de cette différence (la réalité meilleure que la modélisation) n'est pas comprise, sans doute faut-il l'attribuer aux incertitudes.

\section{Passage à la carte des épaisseurs}

Les épaisseurs obtenues le sont le long des profils visibles sur la figure 3a. Ils sont espacés les uns des autres d'environ
$5 \mathrm{~m}$. Pour produire une carte, il convient d'interpoler ces données, et c'est préférentiellement au krigeage, que nous avons eu recours. La surface topographique et la base des scories ont été interpolées séparément, et le volume des scories est ensuite obtenu par intégration 2D de la différence. Sur l'échelle du prospect, le variogramme de la topographie relève d'un modèle linéaire. Celui de la surface basale (mur) de la couche de scorie est affine, avec un petit effet de pépite.

\section{Estimation de l'erreur}

Le krigeage permet d'estimer une erreur formelle. Toutefois, la détermination de l'épaisseur des scories passe par de nombreuses étapes, qui vont du choix de la méthode géophysique au pointage manuel des sections, via l'inversion par RES2DINV. L'erreur est très difficilement estimable avec fiabilité. Elle dépendrait ici de la topographie accidentée, de la densité des scories, malgré tout un peu variable, de choix divers (élimination de certaines mesures aberrantes) etc.

Les géophysiciens savent que les erreurs d'estimation de campagnes géophysique opérées dans les milieux naturels avoisinent souvent les $20 \%$. Cela est valable du moins en l'absence d'a priori forts, et nous pourrions retenir cette valeur en adoptant une posture de prudence. Toutefois, il peut être fait état dans le cas présent d'a priori solides : homogénéité de la couche de scorie, passage brutal au substratum sidérolitique, et leçons tirés des précédentes recherches relativement à la linéarité. Sans calcul dédié (qui s'avérerait difficile), nous pouvons supposer une erreur d'estimation globale deux fois moindre, de l'ordre de $10 \%$

\section{RÉSULTATS}

La figure $3 c$ donne la carte des épaisseurs de scories, résultat de la chaîne de traitement décrite plus haut, tandis que la figure $3 \mathrm{~d}$ montre la topographie de la base des scories. L'image $3 \mathrm{~b}$ montre la topographie en perspective sous la forme d'une grille développée dans l'espace, drapée en 2-D de l'épaisseur des scories (en couleur).

Ces figures montrent, au SSE, un amas principal, où l'épaisseur atteint presque $5 \mathrm{~m}$, tandis qu'une deuxième accumulation plus modeste existe au $\mathrm{NO}$ avec une épaisseur qui ne dépasse pas $3 \mathrm{~m}$. Entre les deux, un épandage peu structuré fait de 1 à $2 \mathrm{~m}$ d'épaisseur au maximum. Notons que la fouille du ferrier test n'a livré que des déchets liés à la réduction. La post-réduction ne se pratiquait a priori pas sur place dans cet atelier.

L'intégration du volume de cette couche (directement faite avec le logiciel Surfer) conduit à une estimation de $2240 \mathrm{~m}^{3}$ 
(à $10 \%$ près si l'on retient l'estimation faite plus haut). C'est environ $40 \%$ de plus que l'estimation initiale de $1600 \mathrm{~m}^{3}$ fondée sur la reconnaissance de surface et au sein des sondages. La densité des scories étant proche de 2, il y aurait là environ 4500 tonnes de scories, qui correspondraient, pour ce ferrier $\mathrm{n}^{\circ} 1$, à une production de fer comprise entre 3200 et 4000 tonnes après application du coefficient de conversion dont le principe est décrit et référencé plus haut (Decombeix et al., 1998.).

\section{ConClusion}

L'étude confirme le potentiel de la méthode de polarisation provoquée pour la caractérisation de sites sidérurgiques anciens. Elle se présente aujourd'hui comme la seule méthode d'estimation des volumes de scories qui soit alternative aux sondages. Elle est non invasive et permet de déterminer la géométrie et in fine le volume de tels amas de résidus.

La réponse en chargeabilité est linéaire par rapport à la concentration de scories. En cela, la polarisation provoquée se distingue des méthodes électriques classiques, ces dernières ne possédant pas cette propriété de linéarité vis-à-vis des structures. Cette linéarité, qui permet après conversion par une loi linéaire ou affine d'estimer le tonnage des scories, se démontre également en considérant que le "signal PP " relève d'une perturbation (au sens de la théorie mathématique des perturbations) de la résistivité.

Ainsi, les deux propriétés que sont d'une part la spécificité de la réponse PP aux scories de métallurgie du fer et d'autre part la linéarité de la réponse par rapport à la concentration aux scories, conduisent à proposer un usage plus systématique de la PP pour ce type d'étude paléométallurgique.

Rappelons toutefois qu'il ne faut pas considérer cette méthode géophysique, pas plus qu'une autre, comme une panacée, qui conduirait à éviter le contrôle et la validation par des sondages. S'il est évident qu'une prospection avant fouille peut conduire à une économie sensible dans l'exploration d'un site, il appartiendra toujours à l'archéologue de jauger les éléments dont il dispose pour prendre ou non une décision de fouille, en profitant d'un faisceau d'arguments dont les résultats de polarisation provoquée ne sont qu'un des multiples rameaux.

Enfin, l'usage de la méthode PP à d'autres types de métallurgie (argent, cuivre) reste à explorer : ce qui est fait ici ne va, pour l'heure que pour le fer, et l'intérêt d'une telle démarche pour la métallurgie des non-ferreux reste à étudier et constitue de fait un sujet de recherche pour l'avenir.

\section{Remerciements}

Le laboratoire UMMISCO, UMI n 209 IRD-UPMC et le Conseil Général de la Dordogne ont, grâce aux financements accordés, permis cette étude. Les étudiants de M1 de l'UPMC: Manon Cassagnole, David Durka et Séréna Delar, ont participé aux opérations de terrain et au traitement des données et nous les en remercions. Nous adressons particulièrement nos remerciements aux deux relecteurs dont le travail a permis de notables améliorations. 


\section{ANNEXE - Fiche descriptive du site dit "Les Grands Bois " et localisation des ferriers, la zone en quadrillé représente les terrains altérés par le défrichage (voir également Bonnefant, 2015a et 2015b).}

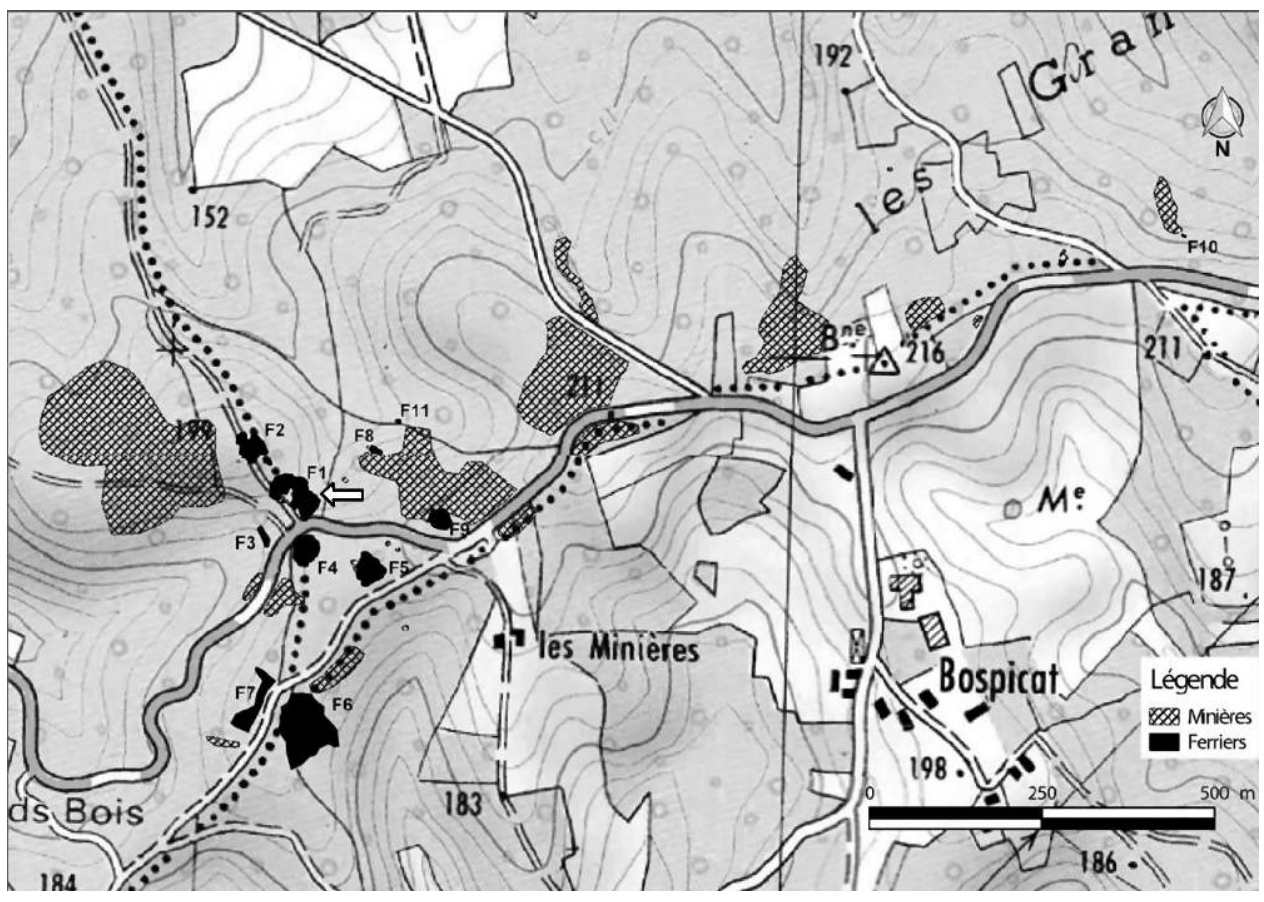

Région : Nouvelle Aquitaine Département Dordogne Commune : Saint-Vincentsur l'Isle

Lieu-dit : Les Grands Bois

Sections et parcelles : B2 411 et B2 326

Coordonnées Lambert 93 : $(\mathrm{X}=534212 ; \mathrm{Y}=6460401)$ et $(X=535493 ; Y=6460784)$ Altitude : 195 et $200 \mathrm{~m}$

Figure 5 : emplacement des ferriers sur le fond topographique, la flèche indiquant le ferrier étudié (les autres ayant été fortement remaniés par des travaux forestiers).

Figure 5: Disposal of the slag heaps (black patches) on the general map. The arrow points the heap we study in this paper. Most of the heaps are already destroyed by forestry activities.

\section{Bibliographie}

Abdulsamad F., Florsch N., Camerlynck C., 2016. Spectral induced polarization in a sandy medium containing semiconductor materials: study of the polarization mechanism. $4^{\text {th }}$ International Workshop on Induced Polarization, Aarhus.

Bertin J., Loeb J., 1976. Experimental and Theoretical aspects of induced polarization, Vol. 1: Presentation and Application of the IP Method; Case Histories, Vol2: Macroscopic and Microscopic Theories. Edited by R. G. van Nostrand and S. Saxov, Schweizerbart Science Publishers, Stuttgart, Germany.

Bonnenfant J., 2015a. Premiers résultats de l'étude du site sidérurgique médiéval des Grands Bois (Dordogne). Documents d'Archéologie et d'Histoire Périgourdines 2014, 29, p. 143-171.

Bonnenfant J., 2015b. Saint-Vincent-sur-l'Isle, les Grands Bois, RFO programmée, SRA Aquitaine.
Decombeix P.-M., Fabre J.-M., Tollon F., Domergue Cl., 1998, Évaluation du volume des ferries romains du domaine des Forges (Les Martys, Aude), de la masse de scories qu'ils renferment et de la production de fer correspondante. Archeosciences, revue d'Archéomètrie, 22, p. 77-90.

Florsch N., Llubes M., Téreygeol F., Ghorbani A., Roblet P., 2011. Quantification of slag heap volumes and masses through the use of induced polarization: application to the Castel-Minier site. Journal of Archaeological Science, 38(2), p. 438-451, DOI:10.1016/j.jas.2010.09.027

Florsch N., Llubes M., Téregeol F., 2012. Induced polarization 3D tomography of an archaeological direct reduction slag heap. Near surface Geophysics, 10, p. 567-574, DOI: 10.3997/1873-0604.2012042.

Fluzin P., Ploquin A., Serneels V., 2000. Archéométrie des déchets de production sidérurgique: Moyens et méthodes 
d'identification des différents éléments de la chaîne opératoire directe. Gallia, 57, p. 101-121 [http://www.jstor.org/ stable/43608164].

IP2016, 2016. $4^{\text {th }}$ International Workshop on Induced Polarization: [http://hgg.au.dk/ip2016/].

Jarrier C., Andrieux Ph., Domergue C., Pieraggi B., Ploquin A., Tollon F., 1997. Élaboration du fer par réduction directe : essais de reproduction des procédés antiques. Rev. Met. Paris, 94(5) , p. 691-704, [http://dx.doi.org/10.1051/ metal/199794050691].

Kemna A., 2000. Tomographic inversion of complex resistivity: theory and application. Onsnabrück: Der Andereverl., 2000. Berichte des Institutes fürGeophysik der Ruhr-Universität Bochum: reihe 1; Nr. 56. Zugl. : Bochum, Der AndereVerlag; Reihe 1; Ruhr-Univ., Diss., 2000. ISBN 3-934366-92-9.

Kemna A., Binley, A., Cassiani, G., Niederleithinger E., Revil A., Slater L., Williams K.H., Orozco A.F., Haegel F.H., Hördt A., Kruschwitz S., Leroux V., Titov K., Zimmermann E., 2012. An overview of the spectral induced polarization method for near-surface applications. Near Surface Geophysics, 10(6), p. 453-468, DOI: 10.3997/18730604.2012027.

Kemna A., Binley A., Slater L., 2004, Crosshole IP imaging for engineering and environmental applications. Geophysics, 69, p. 97-107.

LoKe M. H., 2002. RES2DINV ver. 3.50. Rapid 2-D resistivity and IP inversion using the least square method. Geotomo Software Company.

Loke M. H., Barker R. 2004. RES2Dinv software. Geotomo Software Company.

Olnoeft, 1981. Electrical properties of rocks. In Touloukain, Y.S., Judd, W.R., Roy, R.F. (eds). Physical properties of rocks and mainerals, New York, McGraw Hill.

Mahé-Le Carlier C., Ploquin A., 1999. Typologie et caractérisation des scories de réduction de la métallurgie du fer. Revue d'Archéométrie, 23(1), p. 59-69.

Mao D., Revil A., Hinton J., 2016. Induced polarization response of porous media with metallic particles. - Part 4. Detection of metallic and non-metallic targets in time domain induced polarization tomography. Geophysics, 81(4), p. 359375, DOI: 10.1190/geo2015-0480.1.

Meyer C., Ullrich B., Barlieb C. D.M. (2007). Archaeological questions and geophysical solutions: ground-penetrating radar and induced polarization investigations in Munigua, Spain. Archaeological Prospection, 14, p. 202-212, DOI:10.1002/ arp. 314

Parasnis D.S., 1997. Principle of Applied Geophysics. Chapman \& Hall, London, ISBN 0-412-80250-3.
Pelton W.H., Ward S.H., Hallof P.G., Sill W.R., Nelson P.H., 1978. Mineral discrimination and removal of inductive coupling with multifrequency IP. Geophysics, 43, p. 588-609.

Placencia-Gómez E., Parviainen A., Slater L., Leveinen J., 2015. Spectral induced polarization (SIP) response of mine tailings. Journal of Contaminant Hydrology, 173, p. 8-24, [http:// $d x$. doi.org/10.1016/j.jconhyd.2014.12.002].

Revil A., 2013. Effective conductivity and permittivity of unsaturated porous materials in the frequency range 1 mHz-1GHz. Water Resources Research, 49, p. 306-327, doi:10.1029/2012WR012700, 2013

Revil A., Florsch N., Mao D., 2015a. Induced polarization response of porous media with metallic particles - Part 1: A theory for disseminated semiconductors. Geophysics 80 (5), D525-D538.

Revil A., Abdel Aal G.Z., Atekwana E.A., Mao D., Florsch N., 2015b. Induced polarization response of porous media with metallic particles - Part 2: Comparison with a broad database of experimental data. Geophysics 80 (5), D539-D552.

Schleifer N., Weller A., Schneider S., Junge A., 2002. Investigation of a Bronze Age plankway by spectral induced polarisation. Archaeological Prospection, 9, p. 243-253.

Schlumberger C., 1920. Etude sur la prospection electrique du sous-sol. Gauthier-Villars. Disponible en version numérisée à la BNF [http://catalogue.bnf.frlark:/12148/cb31318371h].

Seigel H.O., Nabighian M., Parasnis D.S., Vozoff K., 2007. The early history of the induced polarization method. Leading Edge, 26, p. 312-321.

SHeEn J., 2016. The Application of Spectral Induced Polarization to Determination of Hydraulic Conductivity. Victoria University of Wellington. Available at: [http://researcharchive.vuw.ac.nz/ xmlui/bitstream/handle/10063/4988/thesis.pdf?sequence=1].

Telford W.M., Geldart L.P., Sheriff R.E., 1990. Applied Geophysics, Cambridge University Press, ISBN 0-521-32693-1.

Ullrich B., Weller A., Günther T., Rücker C., 2007. Geophysical prospecting of ancient slag deposits in Munigua (Spain) and Ain-al Hajer (Morocco) using complex resistivity tomography. $2^{\text {nd }}$ International conference Archaeometallurgy in Europe, Aquileia (Italy) 2007.

Weller A., Brune S., Hennig T., Kansy A., 2000. Spectral induced polarisation at a medieval smelting site. $6^{\text {th }}$ Meeting of Environmental and Engineering Geophysical Society (European Section), Bochum 2000.

Zheng Q., Wei G.W., 2011. Poisson-Boltzmann-Nernst-Planck model. The Journal of Chemical Physics, 134(19), 194101. [http://doi.org/10.1063/1.3581031]. 